Pacific

Journal of

Mathematics

\title{
SYSTEMS OF PARAMETERS AND HOLONOMICITY OF $A$-HYPERGEOMETRIC SYSTEMS
}

Christine Berkesch Zamaere, Stephen Griffeth And EzRa Miller 


\title{
SYSTEMS OF PARAMETERS AND HOLONOMICITY OF $A$-HYPERGEOMETRIC SYSTEMS
}

\author{
Christine Berkesch Zamaere, Stephen Griffeth ANd EZRA Miller
}

\begin{abstract}
We give an elementary proof of holonomicity for $\boldsymbol{A}$-hypergeometric systems, with no requirements on the behavior of their singularities, a result originally due to Adolphson (1994) after the regular singular case by Gelfand and Gelfand (1986). Our method yields a direct de novo proof that $A$-hypergeometric systems form holonomic families over their parameter spaces, as shown by Matusevich, Miller, and Walther (2005).
\end{abstract}

Dedication. Every now and then Andrei Zelevinsky had occasion to write a short and in many ways elementary paper with deep consequences. Particularly close to our hearts are his paper on graded nilpotent classes [Zelevinsky 1985] and his paper with Gelfand and Graev on hypergeometric systems [Gelfand et al. 1987]; both of these had enormous impact on our mathematical careers. It is in that spirit that we dedicate to Andrei this elementary perspective on topics that he influenced substantially for many years.

\section{Introduction}

An $A$-hypergeometric system is the $D$-module counterpart of a toric ideal. Solutions to $A$-hypergeometric systems are functions, with a fixed infinitesimal homogeneity, on an affine toric variety. The solution space of an $A$-hypergeometric system behaves well in part because the system is holonomic, which in particular implies that the vector space of germs of analytic solutions at any nonsingular point has finite dimension.

This note provides an elementary proof of holonomicity for arbitrary A-hypergeometric systems, relying only on the statement that a module over the Weyl algebra in $n$ variables is holonomic precisely when its characteristic variety has dimension at most $n$ (see [Gabber 1981] or [Borel et al. 1987, Theorem 1.12]), along with standard facts about transversality of subvarieties and about Krull

EM had support from NSF grant DMS-1001437. SG acknowledges the financial support of Fondecyt Proyecto Regular 1110072.

MSC2010: primary 33C70; secondary 13N10, 14M25, 16S32, 33C99.

Keywords: hypergeometric, differential operators, toric, holonomic. 
dimension. In particular, our proof requires no assumption about the singularities of the $A$-hypergeometric system; equivalently, the associated toric ideal need not be standard graded. Holonomicity was proved in the regular singular case by Gelfand and Gelfand [1986], and later by Adolphson [1994, §3] regardless of the behavior of the singularities of the system. Adolphson's proof relies on careful algebraic analysis of the coordinate rings of a collection of varieties whose union is the characteristic variety of the system. Another proof of the holonomicity of an A-hypergeometric system, by Schulze and Walther [2008], yields a more general result: for a weight vector $L$ from a large family of possibilities, the $L$ characteristic variety for the $L$-filtration is a union of conormal varieties and hence has dimension $n$. Holonomicity follows when $L=(0, \ldots, 0,1, \ldots, 1)$ induces the order filtration on the Weyl algebra. The $L$-filtration method uses an explicit combinatorial interpretation of initial ideals of toric ideals, which requires a series of technical lemmas.

Holonomicity of $A$-hypergeometric systems forms part of the statement and proof, by Matusevich, Miller, and Walther [2005], that $A$-hypergeometric systems determine holonomic families over their parameter spaces. The new proof of that statement here serves as a model suitable for generalization to hypergeometric systems for reductive groups, in the sense of Kapranov [1998].

The main step (Theorem 1.2) in our proof is an easy geometric argument showing that the Euler operators corresponding to the rows of an integer matrix $A$ form part of a system of parameters on the product $\mathbb{k}^{n} \times X_{A}$, where $\mathbb{k}$ is any algebraically closed field and $X_{A}$ is the toric variety over $\mathbb{k}$ determined by $A$. This observation leads quickly in Section 2 to the conclusion that the characteristic variety of the associated $A$-hypergeometric system has dimension at most $n$, and hence that the system is holonomic. Since the algebraic part of the proof holds when the entries of the parameter $\beta$ are considered as independent variables that commute with all other variables, the desired stronger consequence is immediate: the $A$-hypergeometric system forms a holonomic family over its parameter space (Theorem 2.1).

\section{Systems of parameters via transversality}

Fix a field $\mathbb{k}$. Let $x=x_{1}, \ldots, x_{n}$ and $\xi=\xi_{1}, \ldots, \xi_{n}$ be sets of coordinates on $\mathbb{k}^{n}$ and let $x \xi$ denote the column vector with entries $x_{1} \xi_{1}, \ldots, x_{n} \xi_{n}$. Given a rectangular matrix $L$ with $n$ columns, write $L x \xi$ for the vector of bilinear forms given by multiplying $L$ times $x \xi$.

Lemma 1.1. Let $\mathbb{k}^{2 n}=\mathbb{k}_{x}^{n} \times \mathbb{k}_{\xi}^{n}$ have coordinates $(x, \xi)$ and let $X \subseteq \mathbb{k}_{\xi}^{n}$ be a subvariety. If $L$ is an $\ell \times n$ matrix with entries in $\mathbb{k}$, then the variety $\operatorname{Var}(L x \xi)$ of $L x \xi$ in $\mathbb{k}^{2 n}$ is transverse to $\mathbb{k}^{n} \times X$ at any smooth point of $\mathbb{k}^{n} \times X$ whose $\xi$-coordinates are all nonzero. 
Proof. It suffices to prove the statement after passing to the algebraic closure of $k$, so assume $\mathbb{k}$ is algebraically closed. Let $(p, q)$ be a smooth point of $\mathbb{k}^{n} \times X$ that lies in $\operatorname{Var}(L x \xi)$ and has all coordinates of $q$ nonzero. The tangent space to $\mathbb{k}^{n} \times X$ at $(p, q)$ contains $\mathbb{k}^{n} \times\{0\}$. The tangent space $T_{(p, q)}$ to $\operatorname{Var}(L x \xi)$ is the kernel of the $\ell \times 2 n$ matrix $[L(q) L(p)]$, where $L(p)$ (respectively, $L(q))$ is the $\ell \times n$ matrix that results after multiplying each column of $L$ by the corresponding coordinate of $p$ (respectively, $q$ ). Since the $q$ coordinates are all nonzero, $T_{(p, q)}$ projects surjectively onto the last $n$ coordinates; indeed, if $\eta \in \mathbb{K}_{\xi}^{n}$ is given, then taking $y_{i}=-p_{i} \eta_{i} / q_{i}$ yields $y \in \mathbb{k}_{x}^{n}$ with $L(q) y+L(p) \eta=0$. Thus the tangent spaces at $(p, q)$ sum to the ambient space, so the intersection is transverse.

The next result applies the lemma to an affine toric variety $X$. A fixed $d \times n$ integer matrix $A=\left[\begin{array}{lllll}a_{1} & a_{2} & \cdots & a_{n-1} & a_{n}\end{array}\right]$ defines an action of the algebraic torus $T=\left(\mathbb{k}^{*}\right)^{d}$ on $\mathbb{k}_{\xi}^{n}$ by

$$
t \cdot \xi=\left(t^{a_{1}} \xi_{1}, \ldots, t^{a_{n}} \xi_{n}\right)
$$

The orbit $\operatorname{Orb}(A)$ of the point $\mathbf{1}=(1, \ldots, 1) \in \mathbb{k}^{n}$ is the image of an algebraic map $T \rightarrow \mathbb{k}^{n}$ that sends $t \mapsto t \cdot \mathbf{1}$. The closure of $\operatorname{Orb}(A)$ in $\mathbb{k}^{n}$ is the affine toric variety $X_{A}=\operatorname{Var}\left(I_{A}\right)$ cut out by the toric ideal

$$
I_{A}=\left\langle\xi^{u}-\xi^{v} \mid A u=A v\right\rangle \subseteq \mathbb{k}[\xi]
$$

of $A$ in the polynomial ring $\mathbb{k}[\xi]=\mathbb{k}\left[\xi_{1}, \ldots, \xi_{n}\right]$. The $T$-action induces an $A$ grading on $\mathbb{k}[\xi]$ via $\operatorname{deg}\left(\xi_{i}\right)=a_{i}$, and the semigroup ring $S_{A}=\mathbb{k}[\xi] / I_{A}$ is $A$-graded [Miller and Sturmfels 2005, Chapters 7-8].

For any face $\tau$ of the real cone $\mathbb{R}_{\geq 0} A$ generated by the columns of $A$, write $\tau \preceq A$ and let $\mathbf{1}^{\tau} \in\{0,1\}^{n} \subset \mathbb{k}^{n}$ be the vector with nonzero entry $\mathbf{1}_{i}^{\tau}=1$ precisely when $A$ has a nonzero column $a_{i} \in \tau$. The variety $X_{A}$ decomposes as a finite disjoint union $X_{A}=\bigsqcup_{\tau \preceq A} \operatorname{Orb}(\tau)$ of orbits, where $\operatorname{Orb}(\tau)=T \cdot \mathbf{1}^{\tau}$. Each orbit has dimension $\operatorname{dim} \operatorname{Orb}(\tau)=\operatorname{rank}\left(A_{\tau}\right)$, where $A_{\tau}$ is the submatrix of $A$ consisting of those columns lying in $\tau$, and $\operatorname{dim} X_{A}=\operatorname{rank}(A)$.

Theorem 1.2. The ring $\mathbb{k}[x, \xi] /\left(I_{A}+\langle A x \xi\rangle\right)$ has Krull dimension $n$. In particular, if $A$ has rank $d$ then the forms $A x \xi$ are part of a system of parameters for $\mathbb{k}[x] \otimes_{\mathbb{k}} S_{A}$.

Proof. Let $\mathbb{k}^{\tau} \subseteq \mathbb{k}^{n}$ be the subspace consisting of vectors with 0 in coordinate $i$ if $a_{i} \notin \tau$, and let $|\tau|$ be its dimension. Since $\mathbb{k}[x, \xi] / I_{A}=\mathbb{k}[x] \otimes_{\mathbb{k}} S_{A}$ has dimension $n+\operatorname{rank}(A)$ and the number of $\mathbb{k}$-linearly independent generators of $\langle A x \xi\rangle$ is $\operatorname{rank}(A)$, the Krull dimension in question is at least $n$. Hence it suffices to prove that $\left(\mathbb{k}^{n} \times \operatorname{Orb}(\tau)\right) \cap \operatorname{Var}(A x \xi) \subseteq \mathbb{k}^{n} \times \mathbb{k}^{\tau}$ has dimension at most $n$. Let $x_{\tau}$ and $\xi_{\tau}$ denote the subsets corresponding to $\tau$ in the variable sets $x$ and $\xi$, respectively. The projection of the intersection onto the subspace $\mathbb{k}^{\tau} \times \mathbb{k}^{\tau}$ has image contained in

$$
\left(\mathbb{k}^{\tau} \times \operatorname{Orb}(\tau)\right) \cap \operatorname{Var}\left(A_{\tau} x_{\tau} \xi_{\tau}\right) \subseteq \mathbb{k}^{\tau} \times \mathbb{k}^{\tau}
$$


It therefore suffices to show that the dimension of this latter intersection is at most $|\tau|$. By Lemma 1.1, the intersection is transverse in $\mathbb{k}^{\tau} \times \mathbb{k}^{\tau}$. But the dimension of $\operatorname{Orb}(\tau)$ is the codimension of $\operatorname{Var}\left(A_{\tau} x_{\tau} \xi_{\tau}\right)$ in $\mathbb{k}^{\tau} \times \mathbb{k}^{\tau}$, which completes the proof.

\section{Hypergeometric holonomicity}

In this section, the matrix $A$ is a $d \times n$ integer matrix of full rank $d$. Let

$$
\left.D=\mathbb{C}\langle x, \partial|\left[\partial_{i}, x_{j}\right]=\delta_{i j} \text { and }\left[x_{i}, x_{j}\right]=0=\left[\partial_{i}, \partial_{j}\right]\right\rangle
$$

denote the Weyl algebra over the complex numbers $\mathbb{C}$, where $x=x_{1}, \ldots, x_{n}$ and $\partial_{i}$ corresponds to $\partial / \partial x_{i}$. This is the ring of $\mathbb{C}$-linear differential operators on $\mathbb{C}[x]$.

For $\beta \in \mathbb{C}^{d}$, the $A$-hypergeometric system with parameter $\beta$ is the left $D$-module

$$
M_{A}(\beta)=D / D \cdot\left(I_{A}^{\partial},\left\{E_{i}-\beta_{i}\right\}_{i=1}^{d}\right),
$$

where $I_{A}^{\partial}=\left\langle\partial^{u}-\partial^{v} \mid A u=A v\right\rangle \subseteq \mathbb{C}[\partial]$ is the toric ideal associated to $A$ and

$$
E_{i}-\beta_{i}=\sum_{j=1}^{n} a_{i j} x_{j} \partial_{j}-\beta_{i}
$$

are Euler operators associated to A.

The order filtration $F$ filters $D$ by order of differential operators. The symbol of an operator $P$ is its image $\operatorname{in}(P) \in \operatorname{gr}^{F} D$. Writing $\xi_{i}=\operatorname{in}\left(\partial_{i}\right)$, this means $\operatorname{gr}^{F} D$ is the commutative polynomial ring $\mathbb{C}[x, \xi]$. The characteristic variety of a left $D$-module $M$ is the variety in $\mathbb{A}^{2 n}$ of the associated graded ideal $\operatorname{gr}^{F} \operatorname{ann}(M)$ of the annihilator of $M$. A nonzero $D$-module is holonomic if its characteristic variety has dimension $n$; this is equivalent to requiring that the dimension be at most $n$ (see [Gabber 1981] or [Borel et al. 1987, Theorem 1.12]). The rank of a holonomic $D$-module $M$ is the (always finite) dimension of $\mathbb{C}(x) \otimes_{\mathbb{C}[x]} M$ as a vector space over $\mathbb{C}(x)$; this number equals the dimension of the vector space of germs of analytic solutions of $M$ at any nonsingular point in $\mathbb{C}^{n}$ [Saito et al. 2000, Theorem 1.4.9].

Viewing the $A$-hypergeometric system $M_{A}(\beta)$ as having a varying parameter $\beta \in \mathbb{C}^{d}$, the rank of $M_{A}(\beta)$ is upper semicontinuous as a function of $\beta$ [Matusevich et al. 2005, Theorem 2.6]. This follows by viewing $M_{A}(\beta)$ as a holonomic family [ibid., Definition 2.1] parametrized by $\beta \in \mathbb{C}^{d}$. By definition, this means not only that $M_{A}(\beta)$ is holonomic for each $\beta$, but also that it satisfies a coherence condition over $\mathbb{C}^{d}$ : after replacing $\beta$ with variables $b=b_{1}, \ldots, b_{d}$, the module $\mathbb{C}(x) \otimes_{\mathbb{C}[x]} M_{A}(b)$ is finitely generated over $\mathbb{C}(x)[b]$. (The definition of holonomic family cited above allows sheaves of $D$-modules over arbitrary complex base schemes, but that generality is not needed here.) 
The derivation of the holonomic family property for $M_{A}(b)$ from the holonomicity of the A-hypergeometric system is more or less the same as [ibid., Theorem 7.5], which was phrased in the generality of Euler-Koszul homology of toric modules. The brief deduction here isolates the steps necessary for $A$-hypergeometric systems; its brevity stems from the special status of affine semigroup rings among all toric modules [ibid., Definition 4.5]. Note further that this proof does not require technical combinatorial arguments using standard pairs, as in [Saito et al. 2000]; indeed, in $\left(I_{A}\right)$ need not be a monomial ideal.

Theorem 2.1. The module $M_{A}(b)$ forms a holonomic family over $\mathbb{C}^{d}$ with coordinates $b$. In more detail, as a $D[b]$-module the parametric A-hypergeometric system $M_{A}(b)$ satisfies:

(1) the fiber $M_{A}(\beta)=M_{A}(b) \otimes_{\mathbb{C}[b]} \mathbb{C}[b] /\langle b-\beta\rangle$ is holonomic for all $\beta$; and

(2) the module $\mathbb{C}(x) \otimes_{\mathbb{C}[x]} M_{A}(b)$ is finitely generated over $\mathbb{C}(x)[b]$.

Proof. Since $R=\mathbb{C}[x, \xi] /\left\langle\operatorname{in}\left(I_{A}\right), A x \xi\right\rangle$ surjects onto $\operatorname{gr}^{F} M_{A}(\beta)$, it is enough to show that the ring $R$ has dimension $n$. If $M_{A}(\beta)$ is standard $\mathbb{Z}$-graded (equivalently,

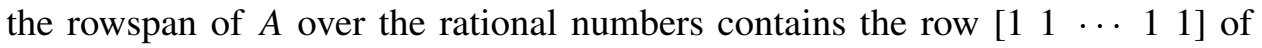
length $n)$, then $\operatorname{in}\left(I_{A}\right)=I_{A} \subseteq \mathbb{C}[\xi]$, and the result follows from Theorem 1.2.

When $M_{A}(\beta)$ is not standard $\mathbb{Z}$-graded, let $\hat{A}$ be the $(d+1) \times(n+1)$ matrix obtained by adding a row of 1's across the top of $A$ and then adding as the leftmost column $(1,0, \ldots, 0)$. If $\xi_{0}$ denotes a new variable corresponding to the leftmost column of $\hat{A}$, and $\hat{\xi}=\left\{\xi_{0}\right\} \cup \xi$, then $\mathbb{C}[\xi] / \operatorname{in}\left(I_{A}\right) \cong \mathbb{C}[\hat{\xi}] /\left\langle I_{\hat{A}}, \xi_{0}\right\rangle$. In particular,

$$
\frac{\mathbb{C}[\hat{x}, \xi]}{\left\langle\operatorname{in}\left(I_{A}\right), A x \xi\right\rangle} \cong \frac{\mathbb{C}[\hat{x}, \hat{\xi}]}{\left\langle I_{\hat{A}}, \xi_{0}, \hat{A} \hat{x} \hat{\xi}\right\rangle},
$$

where $\hat{x}=\left\{x_{0}\right\} \cup x$. Since $\left\langle I_{\hat{A}}, \xi_{0}\right\rangle$ is $\hat{A}$-graded and $\hat{A}$ has a row $\left[\begin{array}{lllll}1 & 1 & \cdots & 1 & 1\end{array}\right]$, we have reduced to the case where $M_{A}(\beta)$ is $\mathbb{Z}$-graded, completing part (1).

With $R$ as in part (1), the ring $R[b]$ surjects onto $\operatorname{gr}^{F} M_{A}(b)$, so it suffices for part (2) to show that $R[b]$ becomes finitely generated over $\mathbb{C}(x)[b]$ upon inverting all nonzero polynomials in $x$. Since the ideal $\left\langle\operatorname{in}\left(I_{A}\right), A x \xi\right\rangle$ has no generators involving $b$ variables, it suffices to show that $R(x)$ itself has finite dimension over $\mathbb{C}(x)$. The desired result follows from the statement proved for part (1): any scheme of dimension $n$ has finite degree over $\mathbb{C}_{x}^{n}$.

\section{References}

[Adolphson 1994] A. Adolphson, "Hypergeometric functions and rings generated by monomials", Duke Math. J. 73:2 (1994), 269-290. MR 96c:33020 Zbl 0804.33013

[Borel et al. 1987] A. Borel, P.-P. Grivel, B. Kaup, A. Haefliger, B. Malgrange, and F. Ehlers, Algebraic D-modules, Perspectives in Mathematics 2, Academic Press, Boston, 1987. MR 89g:32014 Zbl 0642.32001 
[Gabber 1981] O. Gabber, "The integrability of the characteristic variety", Amer. J. Math. 103:3 (1981), 445-468. MR 82j:58104 Zbl 0492.16002

[Gelfand and Gelfand 1986] I. M. Gelfand and S. I. Gelfand, "Generalized hypergeometric equations", Dokl. Akad. Nauk SSSR 288:2 (1986), 279-283. In Russian; translated in Sov. Math., Dokl. 33 (1986), 643-646. MR 87h:22013 Zbl 0634.58030

[Gelfand et al. 1987] I. M. Gelfand, M. I. Graev, and A. V. Zelevinsky, "Holonomic systems of equations and series of hypergeometric type", Dokl. Akad. Nauk SSSR 295:1 (1987), 14-19. In Russian; translated in Soviet Math. Dokl. 36:1 (1988), 5-10. MR 88j:58118 Zbl 0661.22005

[Kapranov 1998] M. Kapranov, "Hypergeometric functions on reductive groups", pp. 236-281 in Integrable systems and algebraic geometry (Kobe/Kyoto, 1997), edited by M.-H. Saito et al., World Sci. Publ., River Edge, NJ, 1998. MR 2000e:33018 Zbl 0987.33008

[Matusevich et al. 2005] L. F. Matusevich, E. Miller, and U. Walther, "Homological methods for hypergeometric families", J. Amer. Math. Soc. 18:4 (2005), 919-941. MR 2007d:13027 Zbl 1095.13033

[Miller and Sturmfels 2005] E. Miller and B. Sturmfels, Combinatorial commutative algebra, Graduate Texts in Mathematics 227, Springer, New York, 2005. MR 2006d:13001 Zbl 1090.13001

[Saito et al. 2000] M. Saito, B. Sturmfels, and N. Takayama, Gröbner deformations of hypergeometric differential equations, Algorithms and Computation in Mathematics 6, Springer, Berlin, 2000. MR 2001i:13036 Zbl 0946.13021

[Schulze and Walther 2008] M. Schulze and U. Walther, "Irregularity of hypergeometric systems via slopes along coordinate subspaces”, Duke Math. J. 142:3 (2008), 465-509. MR 2009b:13067 Zbl 1144.13012

[Zelevinsky 1985] A. V. Zelevinsky, “Two remarks on graded nilpotent classes", Uspekhi Mat. Nauk 40:1(241) (1985), 199-200. In Russian; translated in Russ. Math. Surv. 40:1 (1985), 249-250. MR 86e:14027 Zbl 0577.20032

Received January 10, 2015. Revised February 19, 2015.

CHRISTINE BERKESCH ZAMAERE

SCHOOL OF MATHEMATICS

UNIVERSITY OF MINNESOTA

MINNEAPOLIS, MN 55455

UNITED STATES

cberkesc@math.umn.edu

\section{STEPHEN GRIFFETH}

Instituto de Matemática y Física

UNIVERSIDAD DE TALCA

TALCA

CHILE

sgriffeth@inst-mat.utalca.cl

\section{EZRA MiLlER}

Mathematics Department

DUKE UNIVERSITY

DURHAM, NC 27708

UNITED STATES

ezra@math.duke.edu 


\title{
PACIFIC JOURNAL OF MATHEMATICS
}

\author{
msp.org/pjm
}

Founded in 1951 by E. F. Beckenbach (1906-1982) and F. Wolf (1904-1989)

\section{EDITORS}

Don Blasius (Managing Editor)

Department of Mathematics

University of California

Los Angeles, CA 90095-1555

blasius@math.ucla.edu

\author{
Paul Balmer \\ Department of Mathematics \\ University of California \\ Los Angeles, CA 90095-1555 \\ balmer@math.ucla.edu \\ Robert Finn \\ Department of Mathematics \\ Stanford University \\ Stanford, CA 94305-2125 \\ finn@math.stanford.edu \\ Sorin Popa \\ Department of Mathematics \\ University of California \\ Los Angeles, CA 90095-1555 \\ popa@math.ucla.edu
}

\author{
Vyjayanthi Chari \\ Department of Mathematics \\ University of California \\ Riverside, CA 92521-0135 \\ chari@math.ucr.edu \\ Kefeng Liu \\ Department of Mathematics \\ University of California \\ Los Angeles, CA 90095-1555 \\ liu@math.ucla.edu \\ Jie Qing \\ Department of Mathematics \\ University of California \\ Santa Cruz, CA 95064 \\ qing@ cats.ucsc.edu
}

\section{PRODUCTION}

Silvio Levy, Scientific Editor, production@msp.org

\section{SUPPORTING INSTITUTIONS}

ACADEMIA SINICA, TAIPEI

CALIFORNIA INST. OF TECHNOLOGY

INST. DE MATEMÁTICA PURA E APLICADA

KEIO UNIVERSITY

MATH. SCIENCES RESEARCH INSTITUTE

NEW MEXICO STATE UNIV.

OREGON STATE UNIV.

\author{
STANFORD UNIVERSITY \\ UNIV. OF BRITISH COLUMBIA \\ UNIV. OF CALIFORNIA, BERKELEY \\ UNIV. OF CALIFORNIA, DAVIS \\ UNIV. OF CALIFORNIA, LOS ANGELES \\ UNIV. OF CALIFORNIA, RIVERSIDE \\ UNIV. OF CALIFORNIA, SAN DIEGO \\ UNIV. OF CALIF., SANTA BARBARA
}

\author{
Daryl Cooper \\ Department of Mathematics \\ University of California \\ Santa Barbara, CA 93106-3080 \\ cooper@math.ucsb.edu \\ Jiang-Hua Lu \\ Department of Mathematics \\ The University of Hong Kong \\ Pokfulam Rd., Hong Kong \\ jhlu@maths.hku.hk \\ Paul Yang \\ Department of Mathematics \\ Princeton University \\ Princeton NJ 08544-1000 \\ yang@math.princeton.edu
}

These supporting institutions contribute to the cost of publication of this Journal, but they are not owners or publishers and have no responsibility for its contents or policies.

See inside back cover or msp.org/pjm for submission instructions.

The subscription price for 2015 is US \$420/year for the electronic version, and \$570/year for print and electronic.

Subscriptions, requests for back issues and changes of subscribers address should be sent to Pacific Journal of Mathematics, P.O. Box 4163, Berkeley, CA 94704-0163, U.S.A. The Pacific Journal of Mathematics is indexed by Mathematical Reviews, Zentralblatt MATH, PASCAL CNRS Index, Referativnyi Zhurnal, Current Mathematical Publications and Web of Knowledge (Science Citation Index).

The Pacific Journal of Mathematics (ISSN 0030-8730) at the University of California, c/o Department of Mathematics, 798 Evans Hall \#3840, Berkeley, CA 94720-3840, is published twelve times a year. Periodical rate postage paid at Berkeley, CA 94704, and additional mailing offices. POSTMASTER: send address changes to Pacific Journal of Mathematics, P.O. Box 4163, Berkeley, CA 94704-0163.

PJM peer review and production are managed by EditFLOW ${ }^{\circledR}$ from Mathematical Sciences Publishers.

\section{PUBLISHED BY}

\section{mathematical sciences publishers \\ nonprofit scientific publishing}

http://msp.org/

(C) 2015 Mathematical Sciences Publishers 


\section{PACIFIC JOURNAL OF MATHEMATICS}

Volume $276 \quad$ No. $2 \quad$ August 2015

Free evolution on algebras with two states, II

257

MiCHAEL ANSHELEVICH

Systems of parameters and holonomicity of A-hypergeometric systems

281

Christine Berkesch Zamaere, STEPHEN GRIFfeth and EZRA Miller

Complex interpolation and twisted twisted Hilbert spaces

287

FÉlix Cabello SÁnChez, Jesús M. F. Castillo and Nigel J. Kalton

The ramification group filtrations of certain function field extensions

309

JEFFREY A. CASTAÑEDA and QINGQUAN WU

A mean field type flow, II: Existence and convergence

JEAN-BAPTISTE CASTÉRAS

Isometric embedding of negatively curved complete surfaces in Lorentz-Minkowski space

BING-LONG CHEN and LE YIN

The complex Monge-Ampère equation on some compact Hermitian manifolds

JIANCHUN CHU

Topological and physical link theory are distinct

ALEXANDER COWARD and JOEL HASS

The measures of asymmetry for coproducts of convex bodies

Qi GuO, JinFEng GuO and XunLi Su

Regularity and analyticity of solutions in a direction for elliptic equations

YongYANG Jin, DONGSHENG LI and XU-JIA WANG

On the density theorem for the subdifferential of convex functions on Hadamard spaces

Mina Movahedi, Daryoush Behmardi and Seyedehsomayeh

HOSSEINI

$L^{p}$ regularity of weighted Szegó projections on the unit disc

SAMANGi MunASINGHE and Yunus E. ZEYTUnCU

Topology of complete Finsler manifolds admitting convex functions

SORIN V. SABAU and KatSUHIRO SHIOHAMA

Variations of the telescope conjecture and Bousfield lattices for localized categories of spectra

F. LUKE WOLCOTT 